\title{
Identifying neurological comorbidities in obstructive sleep apnea patients through polysomnography
}

\author{
Lothar Burghaus $^{1,2}$ (1) $\cdot$ Lisa Piano ${ }^{3} \cdot$ Gereon R. Fink $^{2,4} \cdot$ Lennart Knaack $^{3}$ \\ Received: 9 July 2020 / Revised: 12 October 2020 / Accepted: 15 October 2020 / Published online: 22 October 2020 \\ (C) The Author(s) 2020
}

To the Editor,

Obstructive sleep apnea (OSA) is a common disease characterized by recurrent respiratory flow limitations and closure of the upper airway accompanied by repetitive hypoxia during sleep. Increased arousal activity, sleep fragmentation, and disturbed sleep architecture cause cardinal symptoms like excessive daytime sleepiness and impaired quality of life. OSA is associated with an increased risk of complications such as stroke or cardiovascular events. In males with severe OSA, the risk of a cerebrovascular event is nearly three times higher [1]. Likewise, sleep-related breathing and sleep-wake disturbances frequently occur in ischemic stroke. More than $50 \%$ of stroke patients have sleep-related breathing disturbances, presenting with obstructive, central, or mixed apneas [2]. Recently, in this journal, Leino et al. discussed specific polysomnographic features of acute stroke and TIA patients with OSA [3].

Stroke patients are of particular interest because OSA is a significant risk factor for cerebral ischemia, and, vice versa, cerebrovascular lesions can cause sleep disorders [4]. In stroke patients, the treatment of OSA impacts the outcome. Stroke patients with an apnea-hypopnea index $(\mathrm{AHI})>20$ effectively treated by continuous positive airway pressure (CPAP) had a significantly better neurological outcome after 1 month than the control group without CPAP treatment [5]. Consequently,

Lothar Burghaus

lothar.burghaus@cellitinnen.de

1 Department of Neurology, Heilig Geist-Hospital, Grasegger Str. 105, 50737 Cologne, Germany

2 Department of Neurology, University Hospital Cologne, Cologne, Germany

3 Intersom Köln, Center of Sleep Medicine and Sleep Research, Cologne, Germany

4 Inst. of Neuroscience and Medicine (INM-3), Forschungszentrum Juelich, Juelich, Germany stroke patients should be screened for sleep apnea after acute cerebral ischemia to start treatment as soon as possible [4].

We screened a large group of sleep laboratory patients with OSA for neurological comorbidities and looked for abnormalities of polysomnographic parameters that might help to identify neurological comorbidities.

We analyzed 776 patients diagnosed with at least mild OSA (AHI $\geq 5 / h$ ). According to current standards, all patients had a suspected diagnosis of OSA and, therefore, underwent polysomnography. We next compared polysomnographic parameters between patients with OSA who additionally had a history of neurological disease and those who did not have any diagnosed neurological comorbidities. The data were also analyzed for each neurological disease, e.g., cerebral ischemia or neurodegenerative diseases. In a further step, significant differences in the parameters were examined as to whether or not they detected yet undiagnosed neurological comorbidity.

Cerebral ischemia as a comorbidity was found in 27 of the 776 patients (mean age 68.7 years \pm 10.7 versus 56.9 years \pm 12.4). These patients showed particularly poor sleep efficiency and a higher proportion of apnea in the AHI (47.5\% versus 28.7\%). Mean oxygen desaturation index (ODI) was 29.2 ( \pm 18.1 ) and oxygen saturation was lower than $90 \%$ in $9.4 \%$ ( \pm 14.0) of measuring time. Further information on the patients is listed in Table 1.

The key question is , conversely, if patients who suffer from a particularly high proportion of apnea in their AHI and poor sleep efficiency may be those patients that have already suffered from cerebral ischemia. This question should be answered using logistic regression and calculating the odds ratio. The regression coefficient showed $(p=0.004)$ that the lower the relative apnea index based on the total AHI $(\mathrm{OR}=.972)$ and the higher the sleep efficiency $(\mathrm{OR}=$ 1.038), the more likely the patient was not in the group with cerebral ischemia (Fig. 1).

Patients with neurological disorders such as stroke or neurodegenerative diseases should be screened for sleep-related breathing disorders regularly. In this study, we demonstrated 
Table 1 Polysomnographic indices in patients with and without cerebral ischemia

\begin{tabular}{|c|c|c|c|c|}
\hline & \multicolumn{2}{|c|}{ Patients with cerebral ischemia $(n=27)$} & \multicolumn{2}{|c|}{ Control group $(n=749)$} \\
\hline & M & SD & M & SD \\
\hline Age (years) & 68.67 & 10.69 & 56.87 & 12.35 \\
\hline Neck circumference $(\mathrm{cm})$ & 42.15 & 2.60 & 41.66 & 3.85 \\
\hline BMI $\left(\mathrm{kg} / \mathrm{m}^{2}\right)$ & 27.93 & 2.83 & 29.54 & 5.05 \\
\hline AHI & 30.33 & 19.74 & 30.80 & 21.63 \\
\hline AI & 17.67 & 18.95 & 10.72 & 13.47 \\
\hline $\mathrm{HI}^{*}$ & 12.67 & 8.62 & 20.07 & 14.84 \\
\hline $\mathrm{AI} \mathrm{P}^{*}$ & 47.50 & 30.92 & 28.73 & 23.49 \\
\hline HI P* & 52.50 & 30.92 & 71.27 & 23.49 \\
\hline RDI & 34.36 & 18.31 & 35.63 & 20.80 \\
\hline ESS* & 6.26 & 3.16 & 7.65 & 4.67 \\
\hline $\mathrm{O}_{2}$ & 94.60 & 1.99 & 94.28 & 1.68 \\
\hline $\mathrm{O}_{2} \min$ & 82.52 & 6.94 & 82.52 & 7.16 \\
\hline $\mathrm{O}_{2}$ mean & 6.12 & 1.91 & 5.88 & 1.97 \\
\hline PLMI & 27.20 & 25.89 & 18.98 & 23.71 \\
\hline REM\% & 12.60 & 5.63 & 14.85 & 6.73 \\
\hline NREM1\% & 23.38 & 17.31 & 19.65 & 17.51 \\
\hline NREM $2 \%$ & 46.99 & 18.83 & 47.87 & 15.24 \\
\hline NREM3\% & 17.04 & 11.82 & 17.63 & 10.04 \\
\hline REM latency & 145.07 & 64.71 & 13.01 & 82.26 \\
\hline Sleep efficiency* & 74.35 & 14.69 & 81.16 & 11.57 \\
\hline Sleep latency & 34.75 & 49.13 & 20.43 & 24.50 \\
\hline Arousal index & 45.95 & 20.30 & 44.84 & 19.66 \\
\hline SWS-Latency* & 118.67 & 120.14 & 77.92 & 104.50 \\
\hline Snore index & 27.01 & 21.65 & 24.50 & 20.11 \\
\hline
\end{tabular}

$B M I$ body mass index, $A H I$ apnea + hypopnea index, $A I$ apnea index, $H I$ hypopnoea index, $A I-P$ apnoea percentage of total AHI, HI-P hypopnoea percentage of total AHI, RDI respiratory disturbance index, ESS Epworth Sleepiness Scale, $\mathrm{O}_{2}$ oxygen saturation (min: lowest value of oxygen saturation, mean: mean value of oxygen desaturation), PLMI periodic limb movement index, REM rapid eye movement sleep, $S W S$ slow wave sleep, $M$ mean value, $S D$ standard deviation $* p \leq 0.05$

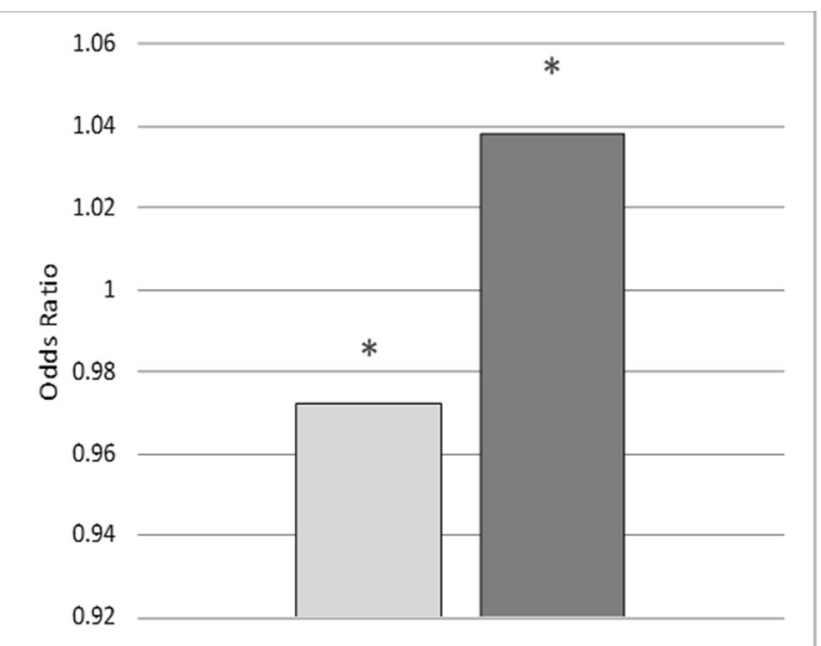

Fig. 1 Logistic regression; independent variables: relative proportion of apnea in total AHI (left column) and sleep efficiency (right column), dependent variable: cerebral ischemia; ${ }^{*} p \leq 0.05$ that vice versa, regular polysomnographic parameters may be indicative of a cerebrovascular disease in patients with OSA. Therefore, we suggest that patients with OSA who present with a high relative apnea index and poor sleep efficiency in standardized polysomnography should be screened for cerebrovascular diseases. To what extent patients with OSA who have polysomnographic findings may benefit from a neurological screening for previously unknown cerebrovascular diseases is the subject of our further research.

Funding Open Access funding enabled and organized by Projekt DEAL.

Open Access This article is licensed under a Creative Commons Attribution 4.0 International License, which permits use, sharing, adaptation, distribution and reproduction in any medium or format, as long as you give appropriate credit to the original author(s) and the source, provide a link to the Creative Commons licence, and indicate if changes were 
made. The images or other third party material in this article are included in the article's Creative Commons licence, unless indicated otherwise in a credit line to the material. If material is not included in the article's Creative Commons licence and your intended use is not permitted by statutory regulation or exceeds the permitted use, you will need to obtain permission directly from the copyright holder. To view a copy of this licence, visit http://creativecommons.org/licenses/by/4.0/.

\section{References}

1. Bassetti CL (2005) Sleep and stroke. Semin Neurol 25(1):19-32. https://doi.org/10.1055/s-2005-867073

2. Hermann DM, Bassetti CL (2009) Sleep-related breathing and sleepwake disturbances in ischemic stroke. Neurology 73(16):13131322. https://doi.org/10.1212/WNL.0b013e3181bd137c

3. Leino A, Westeren-Punnonen S, Töyräs J, Myllymaa S, Leppänen T, Ylä-Herttuala S, Muraja-Murro A, Kantanen AM, Autere J, Jäkälä P,
Mervaala E, Myllymaa K (2020) Acute stroke and TIA patients have specific polygraphic features of obstructive sleep apnea. Sleep Breath 14. https://doi.org/10.1007/s11325-019-02010-2. Online ahead of print

4. Alexiev F, Brill AK, Ott SR, Duss S, Schmidt M, Bassetti CL (2018) Sleep-disordered breathing and stroke: chicken or egg? J Thorac Dis 10:S4244-S4252. https://doi.org/10.21037/jtd.2018.12.66

5. Parra O, Sanchez-Armengol A, Bonnin M, Arboix A, CamposRodriguez F, Perez-Ronchel J, Duran-Cantolla J, de la Torre G, Gonzalez Marcos JR, de la Pena M, Carmen Jimenez M, Masa F, Casado I, Luz Alonso M, Macarron JL (2011) Early treatment of obstructive apnoea and stroke outcome: a randomised controlled trial. Eur Respir J 37(5):1128-1136. https://doi.org/10.1183/ 09031936.00034410

Publisher's note Springer Nature remains neutral with regard to jurisdictional claims in published maps and institutional affiliations. 